\title{
The algebraic dimension of compact complex threefolds with vanishing second Betti number
}

\author{
FRÉDÉRIC CAMPANA ${ }^{1}$, JEAN-PIERRE DEMAILLY ${ }^{2}$ and \\ THOMAS PETERNELL ${ }^{3}$ \\ ${ }^{1}$ Département de Mathématiques, Université de Nancy, BP 239, 54506 Vandoeuvre les Nancy, \\ France \\ ${ }^{2}$ Université de Grenoble I, Institut Fourier, BP 74, U.M.R. 5582 du C.N.R.S., 38402 Saint-Martin \\ d'Heres, France \\ ${ }^{3}$ Mathematisches Institut, Universität Bayreuth, 95440 Bayreuth, Germany; \\ e-mail: thomas.peternell@uni-bayreuth.de
}

(Received: 21 July 1995; accepted in final form 3 April 1997)

\begin{abstract}
This note investigates compact complex manifolds $X$ of dimension 3 with second Betti number $b_{2}(X)=0$. If $X$ admits a non-constant meromorphic function, then we prove that either $b_{1}(X)=1$ and $b_{3}(X)=0$ or that $b_{1}(X)=0$ and $b_{3}(X)=2$. The main idea is to show that $c_{3}(X)=$ 0 by means of a vanishing theorem for generic line bundles on $X$. As a consequence a compact complex threefold homeomorphic to the 6-sphere $S^{6}$ cannot admit a non-constant meromorphic function. Furthermore we investigate the structure of threefolds with $b_{2}(X)=0$ and algebraic dimension 1 , in the case when the algebraic reduction $X \rightarrow \mathbb{P}_{1}$ is holomorphic.
\end{abstract}

Mathematics Subject Classification (1991): 32J17.

Key words: algebraic dimension, algebraic reduction, generic vanishing theorem, topological Euler characteristic

\section{Introduction}

In this note we shall investigate compact complex manifolds of dimension three and second Betti number $b_{2}(X)=0$. Such a manifold cannot be algebraic or Kähler. Therefore we will be interested in the algebraic dimension $a(X)$ which is by definition the transcendence degree of the field of meromorphic functions over the field of complex numbers. Note that $a(X)>0$ if and only if $X$ admits a non-constant meromorphic function. The topological Euler characteristic will be denoted $\chi_{\text {top }}(X)$ which is also the third Chern class $c_{3}(X)$ by a theorem of Hopf. Our main result is

THEOREM. Let $X$ be a compact 3 -dimensional complex manifold with $b_{2}(X)=0$ and $a(X)>0$. Then

$$
c_{3}(X)=\chi_{\text {top }}(X)=2-2 b_{1}(X)-b_{3}(X)=0,
$$

i.e. we either have $b_{1}(X)=0, b_{3}(X)=2$ or $b_{1}(X)=1, b_{3}(X)=0$. 
FRÉDÉRIC CAMPANA ET AL.

Notice that if $a(X)=3$, i.e. $X$ is Moishezon, then we have $b_{2}(X)>0$, but examples of compact threefolds $X$ with $a(X)=1$ or 2 and with the above Betti numbers exist (see Sect. 2).

The following corollary was actually our motivation for the Theorem

COROLLARY. Let $X$ be a compact complex manifold homeomorphic to the 6dimensional sphere $S^{6}$. Then $a(X)=0$.

In other words, $S^{6}$ does not admit a complex structure with a non-constant meromorphic function.

Our Main Theorem is an immediate consequence of the following more general

THEOREM. Let $X$ be a compact 3 -dimensional complex manifold with $b_{2}(X)=0$ and $a(X)>0$. Let $B$ be a vector bundle on $X$. Then $H^{i}(X, B \otimes \mathcal{M})=0$ for $i \geqslant 0$ and generic $\mathcal{M} \in \operatorname{Pic}^{0}(X)$, in particular $\chi(X, B \otimes \mathcal{M})=0$ for all $\mathcal{M} \in \operatorname{Pic}^{0}(X)$.

In the last section we study more closely the structure of threefolds $X$ with $b_{2}(X)=$ 0 and algebraic dimension 1 whose algebraic reduction is holomorphic. We show e.g. that smooth fibers can only be Inoue surfaces, Hopf surface with algebraic dimension 0 or tori.

Finally we would like to thank the referee for suggestions of improvements in the exposition.

\section{Preliminaries and criteria for the vanishing of $H^{0}$}

NOTATIONS 1.0. (1) Let $X$ be a compact complex manifold, always assumed to be connected. The algebraic dimension, denoted $a(X)$, is the transcendence degree of the field of meromorphic functions over $\mathbb{C}$.

(2) $b_{i}(X)=\operatorname{dim} H^{i}(X, \mathbb{R})$ denotes the $i$ th Betti number of $X$.

(3) If $G$ is a finitely generated abelian group, then $\operatorname{rk} G$ will denote its rank (over $\mathbb{Z}$ ).

(4) If $X$ is a compact space, then $h^{q}(X, \mathcal{F})$ denotes the dimension of $H^{q}(X, \mathcal{F})$.

PROPOSITION 1.1. Let $Y$ be a connected compact complex space (not necessarily reduced), every component $Y_{i}$ of $Y$ being of positive dimension. Let $D$ be an effective Cartier divisor on $Y$ such that $D_{\mid Y_{i}} \neq 0$ for all $i$. Let $\mathcal{F}$ be a locally free sheaf on $Y$. Then there exists $k_{0} \in \mathbb{N}$ such that

$$
H^{0}\left(Y, \mathcal{F} \otimes \mathcal{O}_{Y}(-k D)\right)=0
$$

for $k \geqslant k_{0}$.

Proof. We have natural inclusions

$$
H^{0}\left(Y, \mathcal{F} \otimes \mathcal{O}_{Y}(-(k+1) D)\right) \subset H^{0}\left(Y, \mathcal{F} \otimes \mathcal{O}_{Y}(-k D)\right) .
$$

Take $k_{0}$ such that this sequence is stationary for $k \geqslant k_{0}$. Then $s$ has to vanish at any order along $D_{\mid Y_{i}}$ for every $i$ ( $s$ can be thought of locally as a tuple of holomorphic functions), hence $s_{\mid Y_{i}}=0$, and $s=0$. 
COROLLARY 1.2. Let $S$ be a smooth compact complex surface containing an effective divisor $C$ such that $c_{1}\left(\mathcal{O}_{S}(C)\right)=0$. Let $B$ be a vector bundle on $S$. Then for a generic $\mathcal{L} \in \mathrm{Pic}^{0}(X)$ we have

$$
H^{0}(S, B \otimes \mathcal{L})=H^{2}(S, B \otimes \mathcal{L})=0 .
$$

In particular $\chi(S, B)=\chi(S, B \otimes \mathcal{L})=-h^{1}(S, B \otimes \mathcal{L}) \leqslant 0$.

Proof. The vanishing $H^{0}\left(S, B \otimes \mathcal{O}_{S}(-k C)\right)=0$ for large $k$ follows from (1.1). Since $\mathcal{O}_{S}(k C)$ is topologically trivial for large suitable $k$, the required $H^{0}$ vanishing follows from semi-continuity. The $H^{2}$-vanishing follows by applying the previous arguments to $B^{*} \otimes K_{S}$ and Serre duality.

COROLLARY 1.3. Let $X$ be a smooth compact threefold with $b_{2}(X)=0$ carrying an effective divisor $D$. Then $H^{0}(X, B \otimes \mathcal{L})=0$ for generic $\mathcal{L}$ in $\operatorname{Pic}^{0}(X)$ and every vector bundle $B$ on $X$.

Proof. The assumption $b_{2}(X)=0$ means that $H^{2}(X, \mathbb{Z})$ is finite, hence there exists an integer $m>0$ such that $c_{1}\left(\mathcal{O}_{X}(m D)\right)=0$ in $H^{2}(X, \mathbb{Z})$. We can apply (1.1) to obtain

$$
H^{0}\left(X, B \otimes \mathcal{O}_{X}(-k m D)\right)=0
$$

for $k$ large. Now we conclude again by semi-continuity.

The next lemma is well-known; we include it for the convenience of the reader.

LEMMA 1.4. Let $X$ be a compact manifold of dimension $n$ with $a(X)=n$. Then $b_{2}(X)>0$.

Proof. Choose a birational morphism $\pi: \hat{X} \rightarrow X$ such that $\hat{X}$ is a projective manifold. Take a general very ample divisor $\hat{D}$ on $\hat{X}$ and a general curve $\hat{C} \in \hat{X}$. Let $D=\pi(\hat{D})$ and $C=\pi(\hat{C})$. Then $D$ meets $C$ in finitely many points, hence $D \cdot C>0$, in particular $c_{1}\left(\mathcal{O}_{X}(D)\right)$ is not torsion in $H^{2}(X, \mathbb{Z})$.

LEMMA 1.5. Let $X$ be a smooth compact threefold and $f: X \rightarrow C$ be a surjective holomorphic map to a smooth curve $C$. Let $\mathcal{F}$ be a locally free sheaf on $X$. Then $R^{i} f_{*}(\mathcal{F})$ is locally free for all $i$.

Proof. (a) Note that local freeness is equivalent to torsion freeness, since $\operatorname{dim} C=1$. Hence the claim is clear for $i=0$.

(b) Next we treat the case $i=2$. We shall use relative duality (see [RRV71], [We85]); it states in our special situation ( $f$ is flat with even Gorenstein fibers) that if $R^{j} f_{*}(\mathcal{G})$ is locally free for a given locally free sheaf $\mathcal{G}$ and fixed $j$, then

$$
R^{2-j} f_{*}\left(\mathcal{G}^{*} \otimes \omega_{X / C}\right) \simeq R^{j} f_{*}(\mathcal{G})^{*},
$$

in particular $R^{2-j} f_{*}\left(\mathcal{G}^{*} \otimes \omega_{X / C}\right)$ is locally free. Here $\omega_{X / C}=\omega_{X} \otimes f^{*}\left(\omega_{C}^{*}\right)$ is the relative dualizing sheaf. Applying this to $j=0$ and $\mathcal{G}=\mathcal{F}^{*} \otimes \omega_{X / C}^{*}$ our claim for $i=2$ follows. 
(c) Finally we prove the freeness of $R^{1} f_{*}(\mathcal{F})$. By a standard theorem of Grauert it is sufficient that $h^{1}\left(X_{y}, \mathcal{F}_{\mid X_{y}}\right)$ is constant, $X_{y}$ the analytic fiber over $y \in C$. By flatness, $\chi\left(X_{y}, \mathcal{F}_{\mid X_{y}}\right)$ is constant, hence it is sufficient that $h^{j}\left(X_{y}, \mathcal{F}_{\mid X_{y}}\right)$ is constant for $j=0$ and $j=2$. By the vanishing $R^{3} f_{*}(\mathcal{F})=0$, we have (see e.g. [BaSt76])

$$
R^{2} f_{*}(\mathcal{F})_{\mid\{y\}} \simeq H^{2}\left(X_{y}, \mathcal{F}_{\mid X_{y}}\right)
$$

Therefore $h^{2}\left(X_{y}, \mathcal{F}_{\mid X_{y}}\right)$ is constant by (b). Finally

$$
h^{0}\left(X_{y}, \mathcal{F}_{\mid X_{y}}\right)=h^{2}\left(X_{y}, \mathcal{F}_{\mid X_{y}}^{*} \otimes \omega_{X_{y}}\right)=h^{2}\left(X_{y},\left(\mathcal{F}^{*} \otimes \omega_{X}\right)_{\mid X_{y}}\right)
$$

is constant by applying the same argument to $\mathcal{F}^{*} \otimes \omega_{X}$.

\section{The main theorem}

In this section we prove the main result of this note:

THEOREM 2.1. Let $X$ be a 3-dimensional compact complex manifold with $b_{2}(X)=$ 0 and $a(X)>0$. Let $B$ be a vector bundle on $X$. Then

(1) $H^{i}(X, B \otimes \mathcal{M})=0$ for $i \geqslant 0$ and $\mathcal{M} \in \operatorname{Pic}^{0}(X)$ generic.

(2) $\chi(X, B \otimes \mathcal{M})=0$ for all $\mathcal{M} \in \operatorname{Pic}^{0}(X)$.

(3) $c_{3}(X)=0$, i.e. either $b_{1}(X)=0$ and $b_{3}(X)=2$ or $b_{1}(X)=1$ and $b_{3}(X)=0$.

Proof. First notice that (2) and (3) follow from (1). In fact, by (1) we have

$$
\chi(X, B \otimes \mathcal{M})=0
$$

for generic $\mathcal{M}$ and thus the same holds for all $\mathcal{M}$ by Riemann-Roch and the equality $c_{j}(B)=c_{j}(B \otimes \mathcal{M})$. For (3), we apply (2) to $B=T_{X}$ and get

$$
\chi\left(X, T_{X}\right)=0 .
$$

Now, since $H^{2}(X, \mathbb{R})=H^{4}(X, \mathbb{R})=0$, we have $c_{1}(X)=c_{2}(X)=0$, hence

$$
0=\chi\left(X, T_{X}\right)=\frac{1}{2} c_{3}(X)
$$

by Riemann-Roch.

So it suffices to prove (1). Moreover by Serre duality we only need to prove the vanishing for $i=0$ and $i=2$.

In case $i=0$ we observe that there are nonzero effective divisors on $X$ (since $a(X)>0)$ and we can apply (1.3) to get the claim.

So let $i=2$. Let $g: X \_-\mathbb{P}_{1}$ be a nonconstant meromorphic function. Let $\sigma: \hat{X} \rightarrow X$ be a resolution of the indeterminacies of $g$ and let $f: \hat{X} \rightarrow C$ be the 
Stein factorisation of the holomorphic map $\sigma \circ g$. Fix an ample divisor $A$ on $C$ and let $\mathcal{L}$ be the line bundle on $X$ determined by

$$
f^{*}(A)=\sigma^{*}(\mathcal{L}) \otimes \mathcal{O}_{\hat{X}}(-E)
$$

with a suitable effective divisor $E$ supported on the exceptional set of $\sigma$. We need to exhibit a line bundle $\mathcal{M} \in \operatorname{Pic}^{0}(X)$ with

$$
H^{2}(X, B \otimes \mathcal{M})=0 .
$$

We shall distinguish two cases according to whether the indeterminacy locus of $g$ is empty or not.

We start treating the case that $g$ is not holomorphic. First note that the canonical map

$$
H^{2}(X, B \otimes \mathcal{M}) \rightarrow H^{2}\left(\hat{X}, \sigma^{*}(B \otimes \mathcal{M})\right)
$$

is injective. This is obvious from the Leray spectral sequence. Hence it is sufficient to show

$$
H^{2}\left(\hat{X}, \sigma^{*}(B \otimes \mathcal{M})\right)=0 .
$$

Actually for $(*)$ we only need

$$
H^{2}\left(\hat{X}, \sigma^{*}(B \otimes \mathcal{M})(-t E)\right)=0
$$

for some $t \geqslant 0$. To verify that $(* *)$ implies $(*)$, consider the exact sequence

$$
\begin{aligned}
H^{2}\left(\hat{X}, \sigma^{*}(B \otimes \mathcal{M})(-t E)\right) & \rightarrow H^{2}\left(\hat{X}, \sigma^{*}(B \otimes \mathcal{M})\right) \\
& \rightarrow H^{2}\left(t E, \sigma^{*}(B \otimes \mathcal{M})\right)
\end{aligned}
$$

and note that $H^{2}\left(t E, \sigma^{*}(B \otimes \mathcal{M})\right)=0$. This last vanishing is seen as follows: let $A_{t}$ be the complex subspace of $X$ defined by the ideal sheaf $\sigma_{*}(-t E)$, then

$$
H^{2}\left(t E, \sigma^{*}(B \otimes \mathcal{M})\right)=H^{2}\left(A_{t}, B \otimes \mathcal{M}\right)=0
$$

since $\operatorname{dim} A_{t}=1$.

We make the ansatz $\mathcal{M}=\mathcal{L}^{t+k}$ with $t$ and $k$ to be determined; of course we need to prove the vanishing only for one $\mathcal{M}$ by semi-continuity. Using the Leray spectral sequence for $f: \hat{X} \rightarrow C,(* *)$ comes down to

$$
H^{q}\left(C, R^{p} f_{*}\left(\sigma^{*}\left(B \otimes \mathcal{L}^{k}\right)\right) \otimes t A\right)=0
$$

for $p+q=2$, and large $t, k$. For $q=2,(* * *)$ is obvious and for $q=1$ it follows from Serre's vanishing theorem for $t \gg 0$. So let $q=0$. We need to see that

$$
\mathcal{F}=R^{2} f_{*}\left(\sigma^{*}\left(B \otimes \mathcal{L}^{k}\right)\right)=0
$$


Since $\mathcal{F}$ is locally free by (1.5), it suffices that $\mathcal{F}_{\mid F}=0$ for the general fiber $F$ of $f$ and large $k$. But this follows from (1.1), the effective divisor $E_{\mid F}$ being nonzero:

$$
H^{2}\left(F, \sigma^{*}\left(B \otimes \mathcal{L}^{k}\right)\right) \simeq H^{0}\left(F, \sigma^{*}\left(B^{*}\right) \otimes K_{F} \otimes \mathcal{O}_{F}(-k E)\right)=0 .
$$

If $g$ is holomorphic, i.e. we may take $\sigma=\mathrm{id}$, so that $f=g$, this argument does not work since $E=0$. Here we have to replace $\mathcal{L}^{t+k}$ by a different line bundle. First note that

$$
H^{0}\left(C, R^{2} f_{*}(B) \otimes(-t A)\right)=0
$$

for $t$ sufficiently large. We claim that this implies

$$
H^{0}\left(C, R^{2} f_{*}(B \otimes \mathcal{M})\right)=0
$$

for general $\mathcal{M} \in \operatorname{Pic}^{0}(X)$. Let $W=H^{1}\left(X, \mathcal{O}_{X}\right)$. Then every element in $W$ is represented as a topologically trivial line bundle.

Consider locally the universal bundle $\hat{\mathcal{M}}$ on $X \times W$. Let $F=f \times$ id $: X \times W \rightarrow$ $C \times W$ and $\hat{B}=\operatorname{pr}_{X}^{*}(B)$. The coherent sheaf $R^{2} F_{*}(\hat{B} \otimes \hat{\mathcal{M}})$ satisfies

$$
R^{2} F_{*}(\hat{B} \otimes \hat{\mathcal{M}})_{\mid C} \times\{t\} \simeq R^{2} f_{*}\left(B \otimes \hat{\mathcal{M}}_{t}\right)
$$

where $\hat{\mathcal{M}}_{t}$ is the line bundle corresponding to $t \in W$. Choose $m \gg 0$ and $t_{0}$ such that $f^{*}\left(A^{-m}\right)=\hat{\mathcal{M}}_{t_{0}}$. This is possible since $b_{2}(X)=0$. By (+) we have

$$
H^{0}\left(C, R^{2} f_{*}\left(B \otimes \hat{\mathcal{M}}_{t_{0}}\right)\right)=0
$$

Hence it is sufficient to show that $R^{j} F_{*}(\hat{B} \otimes \hat{\mathcal{M}})$ is flat with respect to the projection $q: C \times W \rightarrow W$, over a Zariski open set of $W$, then the usual semi-continuity theorem gives the claim (2). Now $R^{2} f_{*}\left(B \otimes \hat{\mathcal{M}}_{t}\right)$ is locally free on $C=C \times t$ for every $t$ by (1.5), hence it is clear that there is a Zariski open set $U \subset W$ such that $R^{2} F_{*}(\hat{B} \otimes \hat{\mathcal{M}})$ has constant rank over $U$, hence is locally free over $U$ (observe just that the set where the rank of a coherent sheaf is not minimal is analytic). This proves $(++)$.

On the other hand we have for $m \gg 0$ by Serre's vanishing theorem

$$
H^{1}\left(C, R^{1} f_{*}(B) \otimes A^{m}\right)=0 .
$$

In the same way as above we conclude that

$$
H^{1}\left(C, R^{1} f_{*}(B \otimes \mathcal{M})\right)=0
$$

for general $\mathcal{M} \in W$. 
By the Leray spectral sequence we therefore again obtain $H^{2}(X, B \otimes \mathcal{M})=0$ for general $\mathcal{M}$. This finishes the proof of the theorem.

COROLLARY 2.2. Let $X$ be a compact complex threefold homeomorphic to the sphere $S^{6}$. Then every meromorphic function on $X$ is constant.

Proof. Note that $c_{3}(X)=\chi_{\text {top }}\left(S^{6}\right)=2$ and apply (2.1).

Next we give examples of threefolds with $a(X)>0, b_{2}(X)=0$ and $c_{3}(X)=0$ so that the Main Theorem (2.1) is sharp.

EXAMPLE 2.3. The so-called Calabi-Eckmann threefolds are compact threefolds homeomorphic to $S^{3} \times S^{3}$, see [Ue75]. They can be realized as elliptic fiber bundles over $\mathbb{P}_{1} \times \mathbb{P}_{1}$. Hence $a(X)=2, b_{1}(X)=b_{2}(X)=0$ and $b_{3}(X)=2$.

We now show that Calabi-Eckmann manifolds can be deformed to achieve $a(X)=1$ or $a(X)=0$ and $b_{1}=b_{2}=0, b_{3}=2$. We choose positive real numbers $a, b, c$ and let $B=\mathbb{C}^{2} \backslash\{(0,0)\}$. We define the following action of $\mathbb{C}$ on $B \times B$ :

$$
(t, x, y, u, v) \mapsto(\exp (t) x, \exp (a t) y, \exp (i b t) u, \exp (i c t) v) .
$$

One checks easily that this action is holomorphic, free and almost proper so that the quotient $X$ exists and is a compact manifold. If $a=1$ and $b=c$, then $X$ is a Calabi-Eckmann manifold. If however $a \notin \mathbb{Q}$ and $b=c$ resp. $a \notin \mathbb{Q}$ and $\frac{b}{c} \notin \mathbb{Q}$, then $a(X)=1$ resp. $a(X)=0$.

EXAMPLE 2.4. Hopf threefolds of the form

$$
\mathbb{C}^{3} \backslash\{(0,0,0)\} / \mathbb{Z}
$$

with the action of $\mathbb{Z} \simeq\left\{\lambda^{k} ; k \in \mathbb{Z}\right\}$ being defined by

$$
\lambda(x, y, z)=(\alpha x, \beta y, \gamma z), \quad 0<|\alpha|,|\beta|,|\gamma|<1
$$

are homeomorphic to $S^{1} \times S^{5}$. They have $a(X)=0,1$ or 2 and $b_{1}(X)=1$, while $b_{2}(X)=b_{3}(X)=0$. This realizes the other possibility for the pair $\left(b_{1}, b_{3}\right)$ when $b_{2}=0$ and $a(X)>0$, as stated in the Main Theorem.

Notice that the algebraic reduction is holomorphic in (2.3) but it is not holomorphic in (2.4) if $a(X)=1$.

EXAMPLE 2.5. We finally give other examples of compact threefolds $X$ with $a(X)=0$ and $b_{1}=b_{2}=0, b_{3}=2$. Let $\Gamma \subset \operatorname{Si}(2, \mathbb{C})$ be a torsion free cocompact lattice in such a way that the quotient $X:=\operatorname{Si}(2, \mathbb{C}) / \Gamma$ has $b_{1}(X)=0$.

This last condition is not automatic. Let $Y=\operatorname{SU}(2) \backslash \operatorname{Sl}(2, \mathbb{C}) / \Gamma$; then $Y$ is a compact differentiable manifold admitting a differentiable fibration $\pi: X \rightarrow Y$ with $S^{3}$ is fiber. Since $b_{1}(X)=0$, we also have $b_{1}(Y)=0$, hence $b_{2}(Y)=0$ by Poincaré duality. Now the Leray spectral sequence immediately gives $b_{2}(X)=0$, the fibers of $\pi$ being 3 -spheres. $b_{3}(X)=2$ is again clear from the Leray spectral 
sequence. Finally the fact that $X$ does not carry any nonconstant meromorphic function results from [HM83] from which we even deduce that $X$ does not carry any hypersurface (as $X$ is homogeneous).

\section{On the finer structure of threefolds with $b_{2}(X)=0$ and $a(X)=1$}

In this section we investigate more closely threefolds $X$ with $b_{2}(X)=0$ and algebraic dimension 1. By construction, the algebraic reduction $f: X \rightarrow-V$ is a meromorphic map to a normal projective variety, hence $V$ is a nonsingular curve. We claim that $V$ must be rational. In fact, we have $b_{1}(X) \leqslant 1$ by 2.1 (3); on the other hand, for every holomorphic 1 -form $u$ on $V$, the pull-back $f^{*} u$ is a $d$ closed holomorphic 1-form on $X$, thus $b_{1}(X) \geqslant 2$. Therefore $V$ is rational. In this section we restrict ourselves to the case when the algebraic reduction $f: X \rightarrow V$ is holomorphic. The key to our investigations is

THEOREM 3.1. Let $F$ be a general smooth fiber of $f$. Then the restriction $r: H^{1}\left(X, \mathcal{O}_{X}\right) \rightarrow H^{1}\left(F, \mathcal{O}_{F}\right)$ is surjective.

We need some preparations for the proof of (3.1). Let $\Delta \subset V$ be a finite non empty set such that $A=f^{-1}(\Delta) \subset X$ contains all singular fibers of $f$. Let $V^{\prime}=V \backslash \Delta$, and $X^{\prime}=f^{-1}\left(V^{\prime}\right)$ so that $f^{\prime}=f_{\mid X^{\prime}}$ is a smooth fibration. Let $D_{i}$, $1 \leqslant i \leqslant r$ be the irreducible components of $A$ and let $s=$ card $\Delta$ be the number of connected components of $A$. Furthermore we set $t=b_{1}(F)$ where $F$ is the general smooth fiber of $f$. For a noncompact space $Z$ we let $b_{i}(Z)=\operatorname{dim} H_{i}(Z, \mathbb{R})$, whatever this dimension is. We prepare the proof of (3.1) by three lemmas.

LEMMA 3.2. (1) The natural exact sequence of groups

$$
1=\pi_{2}\left(V^{\prime}\right) \rightarrow \pi_{1}(F) \rightarrow \pi_{1}\left(X^{\prime}\right) \rightarrow \pi_{1}\left(V^{\prime}\right) \rightarrow 1
$$

is exact and (non-canonically) split.

(2) $b_{1}\left(X^{\prime}\right)=b_{1}\left(V^{\prime}\right)+b_{1}(F)=s-1+t$.

(3) $r=s-1+t-b_{1}(X)$.

Proof. (1) Since $V^{\prime}$ is a non-compact Riemann surface, $\pi_{1}\left(V^{\prime}\right)$ is a free group of $s-1$ generators and since $V^{\prime}$ is uniformized by either $\mathbb{C}$ or by the unit disc, we have $\pi_{2}\left(V^{\prime}\right)=1$. Hence the exact homotopy sequence of the fibration $f^{\prime}: X^{\prime} \rightarrow V^{\prime}$ gives the exact sequence of groups stated in (1). Since $\pi_{1}\left(V^{\prime}\right)$ is a free group, the sequence splits.

(2) From (1) we deduce that

$$
H_{1}\left(X^{\prime}, \mathbb{Z}\right) \simeq H_{1}\left(V^{\prime}, \mathbb{Z}\right) \oplus H_{1}(F, \mathbb{Z})
$$

Moreover $f_{*}: \pi_{1}(X) \rightarrow \pi_{1}(V)$ is surjective since the fibers of $f$ are connected. Hence (2) follows.

(3) The cohomology sequence with rational coefficients of the pair $(X, A)$ gives 


$$
0=H^{4}(X) \rightarrow H^{4}(A) \rightarrow H^{5}(X, A) \rightarrow H^{5}(X) \rightarrow H^{5}(A)=0 .
$$

By duality we have $H^{5}(X, A) \simeq H_{1}\left(X^{\prime}\right)$ and $H^{5}(X) \simeq H_{1}(X)$. Hence $r=$ $\operatorname{dim} H^{4}(A)=b_{1}\left(X^{\prime}\right)-b_{1}(X)=s-1+t-b_{1}(X)$ by (2), as claimed.

Now choose an integer $m>0$ such that $c_{1}\left(\mathcal{O}_{X}\left(m D_{i}\right)\right)=0$ in $H^{2}(X, \mathbb{Z})$ for all $1 \leqslant i \leqslant r$. Let $\bigoplus \mathbb{Z}\left[m D_{i}\right] \simeq \mathbb{Z}^{r}$ be the free abelian group generated by $m D_{i}$, $1 \leqslant i \leqslant r$, and let $\phi: \bigoplus \mathbb{Z}\left[m D_{i}\right] \rightarrow \operatorname{Pic}^{0}(X)$ be given by sending $D=\sum_{i} a_{i} m D_{i}$ to $\mathcal{O}_{X}(D)$.

LEMMA 3.3. Let $K=\operatorname{Ker} \phi$ and $I=\operatorname{Im} \phi$. Then $\operatorname{rk} K \leqslant s-1$ and $\operatorname{rk} I \geqslant r-s+1$.

Proof. The kernel $K$ consists of all divisors $D$ such that $\mathcal{O}_{X}(D) \simeq \mathcal{O}_{X}$, i.e. such that $D$ is the divisor of a global meromorphic function $h$ on $X$. As $f: X \rightarrow V$ is the algebraic reduction of $X$, there must exist a meromorphic function $\tilde{h}$ on $V$ such that $h=\tilde{h} \circ f$. Now, the divisor $\tilde{D}$ of $\tilde{h}$ has degree 0 and support in $\Delta=\left\{x_{1}, \ldots, x_{s}\right\}$. This implies that $K$ is contained in $f^{*}\left(\operatorname{Pic}^{0}(\Delta)\right)$. As $\operatorname{Pic}^{0}(\Delta) \simeq \mathbb{Z}^{s-1}$, the claim follows.

The last ingredient in the proof of (3.1) is provided by

LEMMA 3.4. The restriction map $\alpha: H^{1}\left(X, \mathcal{O}_{X}\right) \rightarrow H^{1}\left(X^{\prime}, \mathcal{O}_{X^{\prime}}\right)$ is injective.

Proof. The Leray spectral sequences of the fibrations $f: X \rightarrow V$ and $f^{\prime}: X^{\prime} \rightarrow$ $V^{\prime}$ yield a commutative diagram

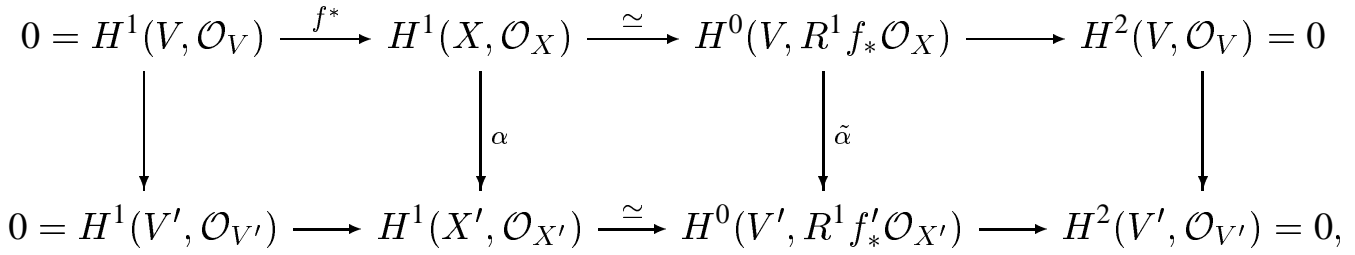

and since $R^{1} f_{*} \mathcal{O}_{X}$ is locally free by (1.5), we conclude that $\tilde{\alpha}$ is injective.

We are now able to finish the proof of (3.1).

Consider again $I \subset \operatorname{Pic}^{0}(X)$, the image of $\phi: \bigoplus \mathbb{Z}\left[m D_{i}\right] \rightarrow \operatorname{Pic}^{0}(X)$, and let $\tilde{I}$ be the inverse image of $I$ under the natural map $H^{1}\left(X, \mathcal{O}_{X}\right) \rightarrow \operatorname{Pic}^{0}(X)$. We have a commutative diagram

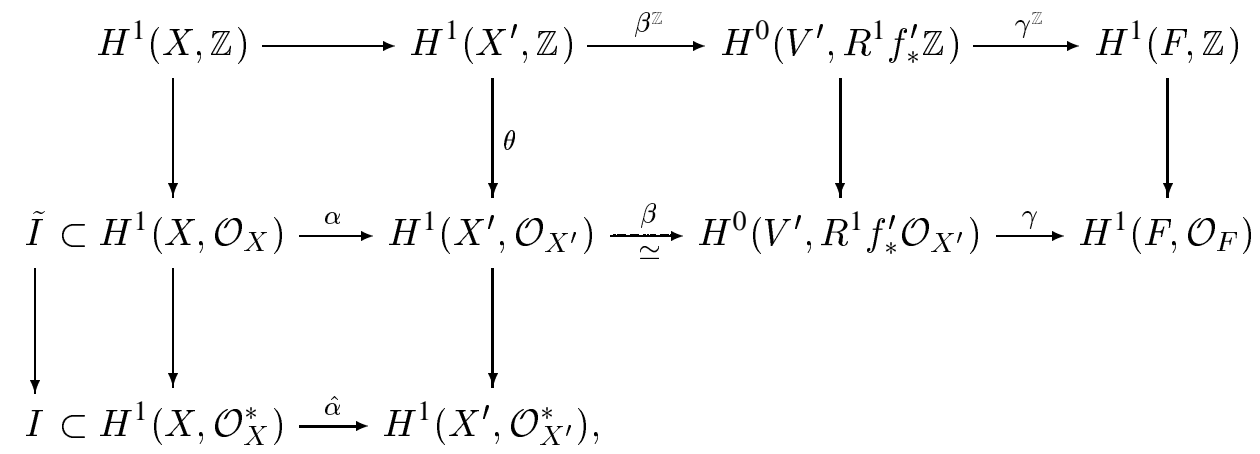


where the two vertical sequences are exponential exact sequences, $\alpha$ and $\hat{\alpha}$ are restriction maps from $X$ to $X^{\prime}, \beta$ and $\beta^{\mathbb{Z}}$ arise from the spectral sequence, and $\gamma$, $\gamma^{\mathbb{Z}}$ are restriction maps to a generic fiber $F$. We get

$$
\operatorname{rk} \tilde{I}=\operatorname{rk} I+\operatorname{rk} H^{1}(X, \mathbb{Z}) \geqslant r-s+1+b_{1}(X)=t
$$

by 3.3 and 3.2 (3). Now $\hat{\alpha}(I)=0$, since $\left.\hat{\alpha}\left(\mathcal{O}_{X}\left(m D_{i}\right)\right)=\mathcal{O}_{X^{\prime}}\left(m D_{i}\right)\right) \simeq \mathcal{O}_{X^{\prime}}$ for all $i$. It follows that $\alpha(\tilde{I})$ is contained in the image of $\theta$, thus $\theta^{-1}(\alpha(\tilde{I})) \subset$ $H^{1}\left(X^{\prime}, \mathbb{Z}\right)$ has rank

$$
\operatorname{rk}\left(\theta^{-1}(\alpha(\tilde{I}))\right) \geqslant \operatorname{rk} \alpha(\tilde{I}) \geqslant \operatorname{rk} \tilde{I} \geqslant t
$$

thanks to the injectivity of $\alpha$. Moreover, $R^{1} f_{*}^{\prime} \mathbb{Z}$ is a locally constant system of rank rk $H^{1}(F, \mathbb{Z})=t$, hence $H^{0}\left(V^{\prime}, R^{1} f_{*}^{\prime} \mathbb{Z}\right)$ has rank at most $t$. On the other hand, as $\beta$ is an isomorphism and $\operatorname{rk} \alpha(\tilde{I}) \geqslant t$, we see that $\beta^{\mathbb{Z}}\left(\theta^{-1}(\alpha(\tilde{I}))\right)$ has rank at least $t$. Therefore, $\gamma^{\mathbb{Z}} \circ \beta^{\mathbb{Z}}\left(\theta^{-1}(\alpha(\tilde{I}))\right)$ is of finite index in $H^{1}(F, \mathbb{Z})$. Since $H^{1}\left(F, \mathcal{O}_{F}\right)$ is the complex linear span of the image of $H^{1}(F, \mathbb{Z})$, we see that $\gamma \circ \beta \circ \alpha(\tilde{I})$ also generates $H^{1}\left(F, \mathcal{O}_{F}\right)$. In particular, the restriction map $H^{1}\left(X, \mathcal{O}_{X}\right) \rightarrow H^{1}\left(F, \mathcal{O}_{F}\right)$ must be surjective. This concludes the proof of (3.1).

(3.5) We now study the structure of the smooth fibers $F$ of $f$. The exact sequence

$$
0 \rightarrow T_{F} \rightarrow\left(T_{X}\right)_{\mid F} \rightarrow \mathcal{O}(F)_{\mid F} \rightarrow 0
$$

and the equality $c_{1}(\mathcal{O}(F))=0$ in $H^{2}(X, \mathbb{R})$ imply

$$
c_{1}(F)=c_{1}(X)_{\mid F}=0, \quad c_{2}(F)=c_{2}(X)_{\mid F}=0
$$

in particular we also have $\chi\left(F, \mathcal{O}_{F}\right)=0$. We conclude from the classification of surfaces that $F$ is one of the following: a Hopf surface, an Inoue surface, a Kodaira surface (primary or secondary), a torus or a hyperelliptic surface (see e.g. [BPV84]). By [Ka69] however, $F$ cannot be hyperelliptic. The reason is the existence of a relative Albanese reduction in that case.

PROPOSITION 3.6. The general fiber $F$ of $f$ cannot be a Kodaira surface nor a Hopf surface with algebraic dimension 1.

Proof. Let $F_{0}$ be a fixed smooth fiber and assume that $F_{0}$ is a Kodaira surface or a Hopf surface with $a\left(F_{0}\right)=1$. Let $g_{0}: F_{0} \rightarrow C_{0}$ be 'the' algebraic reduction which is an elliptic fiber bundle. Let $\mathcal{L}_{0}=g_{0}^{*}\left(\mathcal{G}_{0}\right)$ with $\mathcal{G}_{0}$ very ample on $C_{0}$.

(1) There exists a line bundle $\tilde{\mathcal{L}}$ on $X$ with $\tilde{\mathcal{L}}_{\mid F_{0}}=\mathcal{L}_{0}$.

Proof. By passing to some power $\mathcal{L}_{0}^{m}$ if necessary, we have $c_{1}\left(\mathcal{L}_{0}\right)=0$ in $H^{2}(X, \mathbb{Z})$. Let

$$
\lambda_{1}: H^{1}\left(X, \mathcal{O}_{X}\right) \rightarrow \operatorname{Pic}(X)
$$


and

$$
\lambda_{2}: H^{1}\left(F_{0}, \mathcal{O}_{F_{0}}\right) \rightarrow \operatorname{Pic}\left(F_{0}\right)
$$

be the canonical maps, and let $r: H^{1}\left(X, \mathcal{O}_{X}\right) \rightarrow H^{1}\left(F_{0}, \mathcal{O}_{F_{0}}\right)$ be the restriction map. Choose $\alpha \in H^{1}\left(F_{0}, \mathcal{O}_{F_{0}}\right)$ with $\lambda_{2}(\alpha)=\mathcal{L}_{0}$. Since $r$ is surjective by (3.2), we find $\beta \in H^{1}\left(X, \mathcal{O}_{X}\right)$ with $r(\beta)=\alpha$. Now let $\tilde{\mathcal{L}}=\lambda_{1}(\beta)$.

(2) Let $F$ be any smooth fiber of $f$. Then $\kappa\left(\tilde{\mathcal{L}}_{\mid F}\right)=1$. In fact, it follows from the local freeness of $R^{j} f_{*}(\tilde{\mathcal{L}})$ stated in Lemma 1.5 that

$$
f_{*}\left(\tilde{\mathcal{L}}^{\mu}\right)_{\mid\{y\}} \simeq H^{0}\left(F, \tilde{\mathcal{L}}^{\mu}\right),
$$

where $F=f^{-1}(y)$, see [BaSt76, Chap. 3, 3.10].

(3) From the generically surjective morphism

$$
f^{*} f_{*}\left(\tilde{\mathcal{L}}^{m}\right) \rightarrow \tilde{\mathcal{L}}^{m}
$$

$(m \gg 0)$, we obtain a meromorphic map

$$
g: X \longrightarrow \mathbb{P}\left(f_{*}\left(\tilde{\mathcal{L}}^{m}\right)\right),
$$

which, restricted to $F$ is holomorphic and just gives the algebraic reduction of $F$. Let $Z$ be the closure of the image of $g$. Then $f$ factors via the meromorphic map $h_{1}: X-\rightarrow Z$ and the holomorphic map $h_{2}: Z \rightarrow V$. Now $h_{2}$ is the restriction of the canonical projection $\mathbb{P}\left(f_{*}\left(\tilde{\mathcal{L}}^{m}\right)\right) \rightarrow V$, therefore $h_{2}$ is a projective morphism and $Z$ is projective. Hence $a(X) \geqslant 2$, contradiction.

From (3.6) it follows that $F$ can only be an Inoue surface, a Hopf surface without meromorphic functions or a torus. In order to exclude by a similar method as in (3.6) also tori of algebraic dimension 1, we would need the existence of a relative algebraic reduction (the analogue of $h_{2}: X-\ldots Z$ ) in that case, too.

We now look more closely to the structure of $f$.

PROPOSITION 3.7. Assume that $F$ is not a torus. Then

(1) $R^{1} f_{*}\left(\mathcal{O}_{X}\right)=\mathcal{O}_{V}$

(2) $R^{2} f_{*}\left(\mathcal{O}_{X}\right)=0$

(3) $\operatorname{dim} H^{1}\left(X, \mathcal{O}_{X}\right)=1$,

(4) $H^{2}\left(X, \mathcal{O}_{X}\right)=H^{3}\left(X, \mathcal{O}_{X}\right)=0$.

Proof. (2) Since $H^{2}\left(F, \mathcal{O}_{F}\right)=0$, the sheaf $R^{2} f_{*}\left(\mathcal{O}_{X}\right)$ is torsion, hence identically zero by 1.5 . Then $H^{3}\left(X, \mathcal{O}_{X}\right)=0$ is immediate from the Leray spectral sequence.

(1) Since $h^{1}\left(F, \mathcal{O}_{F}\right)=1, R^{1} f_{*}\left(\mathcal{O}_{X}\right)$ is a line bundle on $V$. Let

$$
d=\operatorname{deg} R^{1} f_{*}\left(\mathcal{O}_{X}\right) .
$$


Then Riemann-Roch gives $\chi\left(R^{1} f_{*}\left(\mathcal{O}_{X}\right)\right)=d+1$. On the other hand the Leray spectral sequence together with $H^{3}\left(X, \mathcal{O}_{X}\right)=0$ and (2) yields

$$
\chi\left(R^{1} f_{*}\left(\mathcal{O}_{X}\right)\right)=h^{1}\left(\mathcal{O}_{X}\right)-h^{2}\left(\mathcal{O}_{X}\right)=-\chi\left(\mathcal{O}_{X}\right)+1 .
$$

We conclude $d=-\chi\left(\mathcal{O}_{X}\right)=0$. This proves (1). Now (3) and the second part of (4) are obvious.

Remark 3.8. In case $F$ is a torus, $R^{1} f_{*}\left(\mathcal{O}_{X}\right)$ is a rank 2 bundle and $R^{2} f_{*}\left(\mathcal{O}_{X}\right)$ is a line bundle. Using Theorem 3.1 it is easy to see that

(1) $R^{1} f_{*}\left(\mathcal{O}_{X}\right)=\mathcal{O}(a) \oplus \mathcal{O}(b)$ with $a, b \geqslant 0$,

(2) $R^{2} f_{*}\left(\mathcal{O}_{X}\right)=\mathcal{O}(a+b)$.

Note that (2) gives dually $f_{*}\left(\omega_{X \mid V}\right)=\mathcal{O}(-a-b)$. Usually one expects the degree of $f_{*}\left(\omega_{X \mid V}\right)$ to be semi-positive, but here we are in a highly non-Kähler situation where it might happen that the above degree is negative, see [Ue87].

PROPOSITION 3.9. Assume that $F$ is not a torus. Then $H^{0}\left(X, \Omega_{X}^{i}\right)=0,1 \leqslant$ $i \leqslant 3$.

Proof. For $i=3$ the claim follows already from 3.7 (4) and Serre duality.

(1) First we treat the case $i=1$. Let $\omega$ be a holomorphic 1-form. Let $j: F \rightarrow X$ be the inclusion. Then $j^{*}(\omega)=0$, hence at least locally near $F$ we have $\omega=f^{*}(\eta)$, hence $\mathrm{d} \omega=0$ near $F$ and therefore the holomorphic 2-form $\mathrm{d} \omega$ is identically zero on $X$. Now the space of closed holomorphic 1 -forms can be identified with $H^{0}\left(X, \mathrm{~d} \mathcal{O}_{X}\right)$ and, as it is well known (see e.g. [Ue75]), we have the inequality

$$
2 h^{0}\left(X, \mathrm{~d} \mathcal{O}_{X}\right) \leqslant b_{1}(X) .
$$

The inequality $b_{1}(X) \leqslant 1$ implies $h^{0}\left(X, \Omega_{X}^{1}\right)=h^{0}\left(X, \mathrm{~d} \mathcal{O}_{X}\right)=0$, as desired.

(2) In case $i=2$, we again have $j^{*}(\omega)=0$. Let $U$ be a small open set in $V$ such that $f_{\mid f^{-1}(U)}$ is smooth. Let $z$ be a coordinate on $U$ and $h=f^{*}(z)$. Then we conclude that

$$
\omega_{\mid f^{-1}(U)}=\mathrm{d} h \wedge \alpha
$$

with some relative holomorphic 1-form $\alpha \in H^{0}\left(f^{-1}(U), \Omega_{X / V}^{1}\right)$. Now again $j^{*}(\alpha)=0$ and therefore $\alpha=0, \omega=0$.

COROLLARY 3.10. Assume that $F$ is not a torus. Then either $f_{*}\left(\Omega_{X / V}^{1}\right)=0$ or there exists some $x \in V$ such that $f_{*}\left(\Omega_{X / V}^{1}\right)=\mathbb{C}_{x}$, i.e. a sheaf supported on $x$ with a 1-dimensional stalk at $x$. In particular $f$ has at most one singular fiber and such a fiber is normal with exactly one singularity of embedding dimension 3.

Proof. Consider the exact sequence

$$
0 \rightarrow f^{*}\left(\Omega_{V}^{1}\right) \rightarrow \Omega_{X}^{1} \rightarrow \Omega_{X / V}^{1} \rightarrow 0 .
$$


Since $F$ has no holomorphic 1-forms, $f_{*}\left(\Omega_{X / V}^{1}\right)$ is a torsion sheaf on the curve $V$. The corollary will follow if we check that $h^{0}\left(V, f_{*}\left(\Omega_{X / V}^{1}\right)\right)=h^{0}\left(X, \Omega_{X / V}^{1}\right) \leqslant 1$. Now, observe the following facts.

(1) $H^{0}\left(X, \Omega_{X}^{1}\right)=0$, by (3.9);

(2) $H^{1}\left(X, f^{*}\left(\Omega_{V}^{1}\right)\right)=H^{1}\left(V, \Omega_{V}^{1}\right)$ by Leray's spectral sequence and the equalities $R^{i} f_{*}\left(f^{*} \Omega_{V}^{1}\right)=R^{i} f_{*}\left(\mathcal{O}_{X}\right) \otimes \Omega_{V}^{1}=\Omega_{V}^{1}, i=0,1$ (cf. 3.7 (1));

(3) $\operatorname{dim} H^{1}\left(V, \Omega_{V}^{1}\right)=1$.

Then, taking cohomology groups in the first exact sequence, we get the desired inequality

$$
h^{0}\left(X, \Omega_{X / V}^{1}\right) \leqslant h^{1}\left(V, \Omega_{V}^{1}\right)=1 .
$$

We can say something more about the structure of the singular fibers of $f$.

PROPOSITION 3.11. Assume that $F$ is not a torus. Let $A$ be a union of fibers containing all singular fibers of $f$. Let $s=\operatorname{card}(f(A))$ and $r$ the number of irreducible components of $A$. Then $r=s$, i.e. all fibers of $f$ are irreducible and $b_{1}(X)=0$.

Proof. Since $F$ is an Inoue surface or a Hopf surface, we have $b_{1}(F)=1$, thus 3.2 (3) implies $r=s-b_{1}(X)$. As $r \geqslant s$, we must have $r=s$ and $b_{1}(X)=0$.

Remark 3.12. In case $F$ is a torus, 3.2 (3) implies $r=s+3-b_{1}(X) \geqslant s+2$. It seems rather reasonable to expect that tori actually cannot appear as fibers of $f$. Observe that $f$ must have a singular fiber in this case because of $r>s$. So a study of the singular fibers is needed to exclude tori as fibers of $f$. However there is a significant difference: the case of tori is one (in fact the only one) where $C_{3,1}$ might fail, see [Ue87].

PROPOSITION 3.13. Assume that $F$ is not a torus. Then $h^{1,1}=h^{1,2}=h^{2,1}=1$ (so that we know all Hodge numbers of $X$ ).

Proof. (1) $h^{1,2}=h^{2,1}$ is of course Serre duality.

(2) By (3.9) and $\chi\left(X, \Omega_{X}^{1}\right)=0$ it suffices to see $h^{1,3}=0$ in order to get $h^{1,1}=h^{1,2}$. But this follows again from Serre duality and the equality $h^{2,0}=$ $h^{0}\left(X, \Omega_{X}^{2}\right)=0$.

(3) From the exact sequence

$$
0 \rightarrow f^{*}\left(\Omega_{V}^{1}\right) \rightarrow \Omega_{X}^{1} \rightarrow \Omega_{X / V}^{1} \rightarrow 0
$$

we deduce that it suffices to show
(a) $h^{2}\left(X, f^{*}\left(\Omega_{V}^{1}\right)\right)=1$,
(b) $h^{2}\left(X, \Omega_{X / V}^{1}\right)=0$,

in order to get $h^{1,2} \leqslant 1$. 
(a) By the Leray spectral sequence and (3.7) we have $h^{2}\left(X, f^{*}\left(\Omega_{V}^{1}\right)\right)=$ $h^{1}\left(V, \Omega_{V}^{1}\right)=1$.

(b) Again we argue by the Leray spectral sequence. Since $R^{1} f_{*}\left(\Omega_{X / V}^{1}\right)$ is a torsion sheaf, we need only to show that

$$
R^{2} f_{*}\left(\Omega_{X / V}^{1}\right)=0
$$

In fact, taking the direct image $f_{*}$ of (S), we see that $R^{2} f_{*}\left(\Omega_{X / V}^{1}\right)$ is a quotient of $R^{2} f_{*}\left(\Omega_{X}^{1}\right)$ which is 0 by the equality $H^{2}\left(F, \Omega_{F}^{1}\right)=H^{0}\left(F, \Omega_{F}^{1}\right)=0$ and by (1.5).

(4) We finally show $h^{1,1} \neq 0$ to conclude the proof. Let $\left(E_{r}^{p, q}\right)$ be the Frölicher spectral sequence on $X$. Since $b_{1}(X)=0$ by (3.11), we get $E_{\infty}^{0,1}=0$. Hence $E_{2}^{0,1}=0$. On the other hand

$$
E_{2}^{0,1}=\operatorname{Ker} \partial: E_{1}^{0,1} \rightarrow E_{1}^{1,1}
$$

Since $E_{1}^{p, q}=H^{p, q}(X)$, we conclude that $H^{1}\left(X, \mathcal{O}_{X}\right)$ injects into $H^{1,1}(X)$. So by (3.7) $H^{1,1}(X) \neq 0$.

We finally collect all our knowledge in the case the general fiber of $f$ is not a torus.

THEOREM 3.14. Let $X$ be a smooth compact threefold with $b_{2}(X)=0$ and holomorphic algebraic reduction $f: X \rightarrow V$ to the smooth curve $V$. Assume that the general smooth fiber is not a torus. Then:

(1) $b_{1}(X)=0, b_{3}(X)=2$.

(2) Any smooth fiber of $f$ is a Hopf surface without meromorphic functions or an Inoue surface.

(3) The Hodge numbers of $X$ are as follows: $h^{1,0}=0, h^{0,1}=0, h^{2,0}=0$, $h^{1,1}=1, h^{0,2}=0, h^{3,0}=0, h^{2,1}=1$ (the others are determined by these via Serre duality).

(4) All fibers of $f$ are irreducible. There is at most one normal singular fiber.

\section{References}

[BaSt76] Banica, C. and Stanasila, O.: Algebraic methods in the global theory of complex spaces. Wiley, 1976.

[BPV84] Barth, W., Peters, C. and van de Ven, A.: Compact complex surfaces. Erg. d. Math. (3), Band 4, Springer, 1984.

[HM83] Huckleberry, A. T. and Margulis, G.: Invariant analytic hypersurfaces. Inv. Math. 71 (1983) 235-240.

[Ka69] Kawai, S.: On compact complex analytic manifolds of complex dimension 3, II. J. Math. Soc. Japan 21 (1969) 604-616.

[RRV71] Ramis, J., Ruget, G. and Verdier, J.-L.: Dualité relative en géométrie analytique complexe. Inv. Math. 13 (1971) 261-283.

[Ue75] Ueno, K.: Classification theory of algebraic varieties and compact complex spaces. Lecture Notes in Math. 439, Springer, 1975. 
[Ue87] Ueno, K.: On compact analytic threefolds with non-trivial Albanese tori. Math. Ann. 278 (1987) 41-70.

[We85] Wehler, J.: Der relative Dualitätssatz für Cohen-Macaulay Räume. Schriftenreihe des Math. Instituts der Univ. Münster, 2. Serie, Heft 35 (1985). 